\title{
Hepcidin/Ferritin Quotient Helps to Predict Spontaneous Recovery from Iron Loss following Blood Donation
}

\author{
Ramin Lotfi ${ }^{a}, b \quad$ Christine Kroll ${ }^{b}$ Dietmar Plonné ${ }^{c}$ Bernd Jahrsdörfer ${ }^{a, b}$ \\ Hubert Schrezenmeier ${ }^{a}$ b \\ a Institute for Transfusion Medicine, University Hospital UIm, UIm, Germany; \\ ${ }^{b}$ Institute for Clinical Transfusion Medicine and Immunogenetics Ulm, German Red Cross Blood Services Baden-Württemberg-Hessen, \\ Ulm, Germany; \\ ${ }^{\mathrm{c}}$ MVZ Humangenetik UIm, Abteilung Laboratoriumsmedizin, Ulm, Germany
}

\section{Keywords}

Hematology · Red cells · Donors .

Health research methodology

\section{Summary \\ Background: Iron supplementation is generally recom- mended for blood donors even though there are inter- individual differences in iron homeostasis. Methods: Fer- ritin levels of repeat donors were compared with first- time donors, retrospectively. Prospectively, we tested 27 male repeat donors for the following parameters at the day of blood donation as well as $1,3,7,10$, and 56 days thereafter: ferritin, hepcidin, transferrin, transferrin re- ceptor, hemoglobin, erythropoietin, reticulocytes, hemo- globin in reticulocyte, twisted gastrulation protein ho- molog 1, and growth differentiation factor-15. Results: 56 days after blood donation, donors' average ferritin dropped to $55 \%$ (range $30-100 \%$ ) compared to the initial value. Of all tested parameters hepcidin showed the highest and most significant changes beginning 1 day after donation and lasting for the whole period of 56 days. Along with ferritin, there was a high variation in hepcidin levels indicating inter-individual differences in hepcidin response to iron loss. Donors with a hepcidin/ ferritin quotient $<0.3$ regained $60 \%$ of their initial ferritin after 56 days, while those with a quotient $\geq 0.3$ reached less than $50 \%$. Conclusion: As hepcidin appears to inte- grate erythropoietic and iron-loading signals, clinical measurement of hepcidin (together with the hepcidin- ferritin ratio) may become a useful indicator of erythro- poiesis and iron kinetics.}

(C) 2015 S. Karger GmbH, Freiburg

\section{Introduction}

Determining hemoglobin $(\mathrm{Hb})$ levels prior to blood donation is standard in most countries in order to protect blood donors from becoming critically anemic. Hb levels of prospective blood donors are usually measured in capillary blood obtained by finger pricking. Regardless of the fact that the reliability of capillary $\mathrm{Hb}$ estimation is discussed controversially [1-4], subclinical iron deficiency (SID) of blood donors cannot completely be excluded by measurement of $\mathrm{Hb}$ levels. About 200-250 mg of iron is lost with each blood donation [5]. Individuals with plasma ferritin levels smaller than $15 \mathrm{ng} / \mathrm{ml}$ are considered having SID [6]. Individuals with SID do not show clinical signs of iron deficiency while having empty iron stores making them prone to develop clinically apparent iron deficiency syndrome including anemia. SID is present in about $10 \%$ of individuals who meet the $\mathrm{Hb}$ criteria for blood donation [6]. Oral iron supplementation is generally recommended for subjects with frequent blood donations and for patients donating autologous blood prior to surgical interventions, even though autologous blood donation has become a rare procedure performed in less than $1 \%$ of cases. However, the term frequent blood donation is not well defined and the efficacy of such a treatment is rarely monitored. In a study of individuals donating a mean of 15 units of blood over 3.5 years, a beneficial effect of oral iron supplementation on iron stores showed significant inter-individual differences [7]. Thus there is a need to determine the two groups of donors: One group of donors might benefit from oral iron substitution, another group, in contrast, need rather longer interdonation intervals. We aimed to address this issue by trying to define an algorithm which could help to distinguish between the two groups. Therefore, we analyzed plasma concentration of factors which are involved in erythropoiesis and iron homeostasis, such as erythro-

\section{KARGER \\ Fax +497614520714

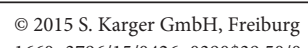


poietin (EPO), ferritin, iron transport protein transferrin (Trf) and its receptor (Trf-R), hepcidin, growth differentiation factor-15 (GDF-15), and twisted gastrulation protein homolog 1 (TWSG 1).

EPO rapidly rises in anemia as a result of anemia-induced hypoxia and falls as $\mathrm{Hb}$ is corrected [8].

Ferritin is a ubiquitous intracellular protein that stores iron and releases it in a controlled fashion. Plasma ferritin is also an indirect marker of the total amount of iron stored in the body; hence serum ferritin is used as a diagnostic test for iron deficiency.

Hepcidin is a liver-derived hormone that regulates iron absorption and recycling via its effects on ferroportin, the cellular iron export protein [9]. Low hepcidin preserves ferroportin and permits increased intestinal iron absorption and enhanced macrophage iron release (and hence iron recycling), whereas elevated hepcidin causes degradation of ferroportin, thus decreasing iron absorption and recycling. Hepcidin is suppressed by erythropoiesis, hypoxia, and iron deficiency and is elevated by iron loading and inflammation [13-17].

Even though EPO significantly decreases serum hepcidin levels in humans, it does not appear to be a direct regulator of hepcidin but acts through erythroferrone (ERFE) which is a hormone produced by erythroblasts

GDF-15 and TWSG1 have been identified through transcriptome analysis as putative erythroblast-derived factors that modulate hepcidin. Recombinant GDF-15 inhibits expression of hepcidin in hepatic cell lines. Expression of GDF-15 peaks in late erythroblasts and is also associated with erythroblast apoptosis. Based on studies in patients with beta-thalassemia and other iron-loading anemias and supported by related animal models and cell-based in vitro studies, it has been proposed that EPO-stimulated erythroblasts secrete mediators that act on the liver to suppress hepcidin production. GDF-15 and TWSG1 have been proposed as a hepcidin suppressor in beta-thalassemia [21,23].

Trf- $\mathrm{R}$ is expressed by erythroblasts showing an increased expression of Trf- $\mathrm{R}$ from early to intermediate stages and a decreasing one with maturation. Thus, Trf-R mass reflects number of immature erythroid cells

Divergent patterns of EPO, soluble Trf-R, and GDF-15 may reflect differing physiologies of these parameters and provide insights into erythropoiesis and iron homeostasis: EPO closely reflects anemia but may also reflect erythroid suppression. Soluble Trf-R reflects overall erythropoietic activity but not necessarily ineffective erythropoiesis or dyserythropoiesis with apoptosis, which appears best reflected by GDF-15.

Given that liver diseases impact iron homeostasis and erythropoiesis by influencing the expression and plasma concentration of hepcidin, EPO, ferritin, and transferrin, liver enzymes (alanine aminotransferase (ALT), aspartate aminotransferase (AST)) need to be tested when measuring these parameters.

Renal excretion plays a major role in clearance of hepcidin. Consistently, patients with chronic kidney diseases have higher hepcidin plasma levels. EPO is expressed in kidney, thus patient with kidney diseases show inadequate EPO concentrations. For these reasons, it is necessary to assess plasma creatinine concentration when measuring EPO and hepcidin.
Plasma levels of ferritin and hepcidin change during infection and inflammation; thus leukocyte count and the concentration of $\mathrm{C}$-reactive protein (CRP) have to be tested concurrently in order to avoid obtaining biased results for ferritin and hepcidin.

In our study we aimed to provide a tool (a single parameter or an algorithm) helping to better estimate recovery from iron loss following blood donation and to better indicate donors profiting from oral iron supplementation in contrast to those who would rather need longer interdonation intervals in order to cope with iron loss. The hepcidin/ferritin quotient here presented qualifies for such an estimation.

\section{Material and Methods}

\section{Human Subjects}

The study was conducted according to the principles expressed in the Declaration of Helsinki. The study was approved by the Institutional Review Board (Ethical Committee) of Ulm University. All blood donors provided written informed consent for the blood donation and the collection of samples.

The retrospective part of the study on ferritin levels was conducted on 1,664 male first-time donors (representing general population) and 5,073 male repeat donors with a history of at least 10 blood donations. Donors with plasma ferritin levels below $15 \mathrm{ng} / \mathrm{ml}$ were considered having SID.

The prospective part of the study was performed on 27 male non-vegetarian healthy regular blood donors without any bleeding events (i.e. blood donations or surgical treatments) within the previous 4 months. Donors were instructed not to change their dietary habits and, specifically, not to take any oral iron supplements.

With regard to the fact that inflammation affects iron homeostasis including concentrations of hepcidin and ferritin, data from donors with CRP levels higher than $5 \mathrm{mg} / \mathrm{ml}$ or leukocyte count higher than $10 \times 10^{3} / \mu \mathrm{l}$ were excluded from the analysis.

\section{Blood Samples}

Blood samples $(10 \mathrm{ml})$ were taken at the day of blood donation (day 0 ) as well as $1,3,7,10$, and 56 days thereafter in order to measure the following parameters: red cell count, leukocytes (including subtypes), platelet count, $\mathrm{Hb}$, reticulocytes (Reti), Hb in reticulocytes (Hb-Reti), EPO, ferritin, Trf, Trf-R, TWSG1, GDF-15, hepcidin, CRP, ALT, AST, and creatinine.

Enzyme-linked immunosorbent assays (ELISA) for GDF-15 (R\&D Systems, Wiesbaden-Nordenstadt, Germany), TWSG1 (Hölzel Diagnostika, Köln, Germany), and hepcidin 25 (DRG Diagnostics, Marburg, Germany) were performed according to the manufacturers' instructions and using a microplate reader (POLARstar Omega, BMG Labtech, Ortenberg, Germany) to measure absorbance. Blood cell count was measured using the automated blood analyzer CELL-DYN (Abbott, Wiesbaden, Germany).

CRP, ALT, AST, and Trf levels were assessed on ABX pentra 400 (HORIBA Medical, Axon Lab AG, Germany) using reagents from DiaSys Greiner GmbH (Flacht, Germany). Ferritin was measured using Architect i1000 SR and reagents from Abbott (Wiesbaden, Germany) according to manufacturer's instructions.

EPO was measured on DXI800i (Beckman Coulter, Krefeld, Germany) using Access EPO Assay from Beckman Coulter according to manufacturer's instructions. Reti-Hb was assessed on hematology analyzer XE-2100 (Sysmex Europe GmbH, Norderstedt, Germany).

\section{Statistics}

Pearson's product-moment correlation and Student's t-test were calculated using R Statistical Software, p values $\leq 0.05$ were considered statistically significant. 


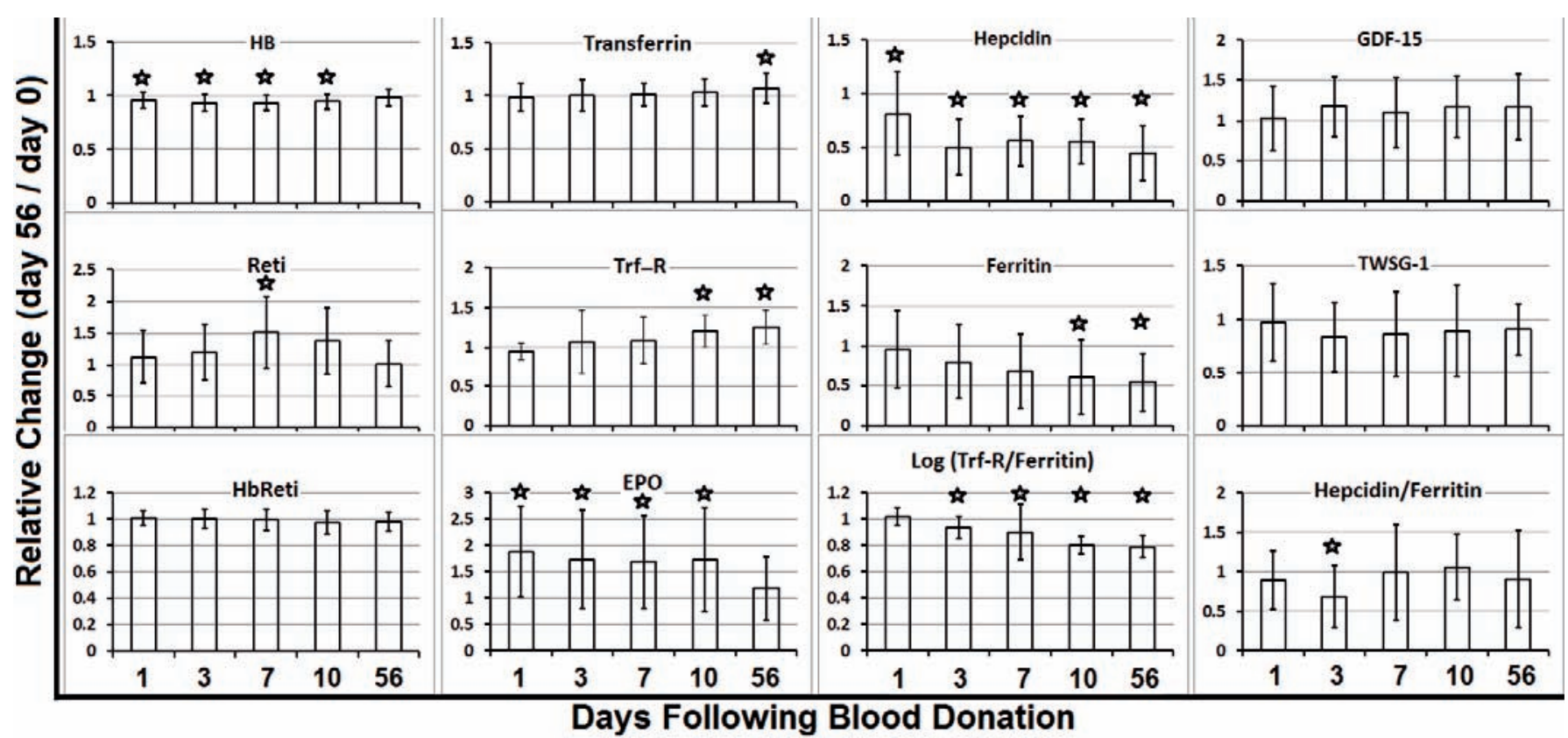

Fig. 1. Two months are not sufficient to spontaneously recover from iron depletion due to blood donation. Indicated parameters were measured in donors' plasma on the day of blood donation (day 0 ) as well as $1,3,7,10$, and 56 days thereafter. The quotient dx/d0 indicates the relative change of measured parameters. Asterisks indicate significant changes in comparison to day 0.

\section{Results}

In our retrospective study on 6,737 male donors, the average ferritin plasma concentration of 1,664 first-time donors (representing general population) was $107.9 \mathrm{ng} / \mathrm{ml}(\mathrm{SD}=76.2)$ with $1.3 \%$ of individuals presenting SID (i.e. ferritin levels below $15 \mathrm{ng} / \mathrm{ml}$ ). On the other hand and consistent with published data $[6,26], 15 \%$ of repeat donors $(n=5,073)$ with a history of at least 10 blood donations presented with SID; the average ferritin level of this population was significantly lower being $49.63 \mathrm{ng} / \mathrm{ml}$ ( $\mathrm{SD}=46.5)$.

The prospective part of the study was performed on 27 male donors with an average age of 34 years (range 19-55 years, mode 22 years) and an average body mass index of $26 \mathrm{~kg} / \mathrm{m}^{2}\left(21-32 \mathrm{~kg} / \mathrm{m}^{2}\right.$, mode $25 \mathrm{~kg} / \mathrm{m}^{2}$ ).

There were no clinical or laboratory signs of infection, inflammation, and liver or kidney diseases with normal values for CRP, and leukocyte count, ALT, AST, and creatinine.

All donors met the $\mathrm{Hb}$ criteria for blood donation with an average predonation Hb level of 159 g/l (134-185 g/l, mode 161 g/l). Within this group, predonation SID was present in 4 individuals (15\%) with ferritin levels ranging from 3.5-11.8 $\mathrm{ng} / \mathrm{ml}$ resulting in an average of $7.5 \mathrm{ng} / \mathrm{ml}$.

The average plasma ferritin concentration of donors without SID $(n=23)$ dropped to $55 \%$ compared to initial values (i.e. before blood donation) 56 days after blood donation (fig. 1). These results were consistent with published data $[26,27]$, indicating that 56 days may not be sufficient to recover from iron depletion due to blood loss, if iron supplementation is not provided.

One day after blood donation (day 1), EPO reached its maximum (190\% compared to baseline level on day 0), stayed at significantly higher levels until day 10 , and declined to $120 \%$ on day 56 . Accordingly, reticulocytes increased to $150 \%$ on day 7 and normalized until day 56 when $\mathrm{Hb}$ levels also reached their initial values (fig. 1), all reflecting normal regulation of erythropoiesis in our study group.

Consistent with the increased number of reticulocytes at day 7 , plasma concentration of Trf- $\mathrm{R}$ - which is expressed on erythroblasts - raised constantly and reached significance 10 days after blood donation (fig. 1). Thus, Trf-R did not qualify for an early predictor. Similarly, average Trf levels reached significant changes not earlier than 56 days and was just 10\% compared to its average initial value(data not shown).

No statistical significant changes could be seen for Hb-Reti, GDF-15, and TWSG1 (fig. 1).

Of all tested parameters hepcidin showed the highest and most significant changes beginning 1 day after blood donation and lasting for the whole period of 56 days. Average ferritin and hepcidin plasma concentrations decreased constantly with ferritin levels reaching statistically significant low levels not earlier than 10 days, while the drop in plasma hepcidin concentrations became statistically significant at day 1 , already (fig.1).

Even though the decrease in donors' average ferritin was significant $(p=0.02)$, we assessed high inter-individual differences in recovery from iron loss, when comparing the ferritin quotient day 56 / day 0 (d56/d0) ranging from 30 to $100 \%$ (average 55\%). Thus, we compared the group of donors with ferritin recovery $<55 \%$ with those having reached $\geq 55 \%$ of their initial ferritin values after 56 days. For the mentioned two subgroups, neither hepcidin level on day 3 by itself nor ferritin level at day 3 qualified as an indicator to be able to predict which donors would recover best from iron loss (fig. 2). 


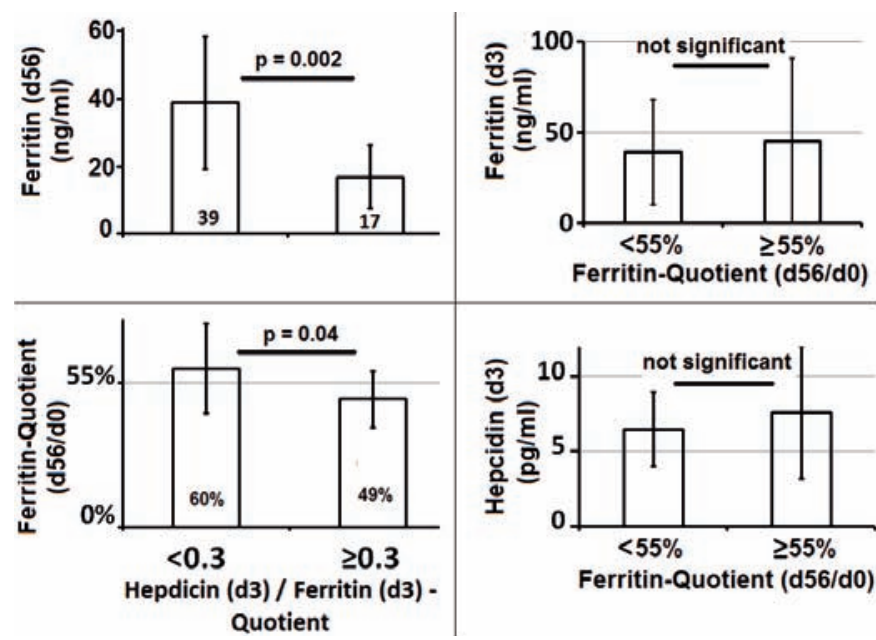

Fig. 2. Hepcidin/ferritin quotient on day 3 helps to predict spontaneous recovery from iron loss. hepcidin/ferritin quotient 3 days after blood donation was correlated with ferritin level on day 56 as well as with ferritin change indicated by the ferritin quotient $\mathrm{d} 56 / \mathrm{fd} 0$. Donors $(\mathrm{n}=17)$ who showed a hepcidin/ ferritin quotient $<0.3$ three days after blood donation recovered significantly better than subjects $(n=10)$ with a quotient $\geq 0.3$.

However, hepcidin levels showed a significant correlation with absolute ferritin levels at day $56(\mathrm{p}=0.001, \mathrm{r}=0.5)$ as well as with ferritin quotient $\mathrm{d} 56 / \mathrm{d} 0(\mathrm{p}=0.05, \mathrm{r}=0.3)$.

We therefore hypothesized that in donors who recover best, hepcidin levels may adapt more rapidly to decreasing ferritin concentrations. Based on this hypothesis, we built the hepcidin/ferritin quotient on day 3 after blood donation and compared donors with above average ferritin recovery with those who showed a rather weak ferritin increase (i.e. below average).

Donors' average hepcidin/ferritin quotient (representing hepcidin response to 'iron loss' or 'need for iron') reached lowest levels on day 3 and differed significantly from values on day 0 (fig. 1).

Donors with a hepcidin/ferritin quotient $<0.3$ showed a significantly higher average ferritin level on day 56 and regained on average $60 \pm 17 \%$ of their initial ferritin after 56 days, while those with a quotient $\geq 0.3$ reached less than $50 \%$ ( $49 \pm 11 \%$ ) (fig. 2 ).

Furthermore, on day 0 the hepcidin/ferritin quotient of donors with SID (i.e. predonation ferritin levels below $15 \mathrm{ng} / \mathrm{ml}$ ) was 1.6, while donors without SID (rest of the donors) showed a significantly $(\mathrm{p}=0.002)$ lower quotient of 0.4 .

Consistently, on day 3 after donation (meaning additional iron deprivation) the average hepcidin/ferritin quotient of primarily iron-deficient donors was $0.5(\mathrm{SD}=0.2)$ and differed significantly $(\mathrm{p}=0.001)$ from the rest of donors showing an average quotient of $0.2(\mathrm{SD}=0.1)$.

Even though GDF-15 and hepcidin values on day 3 significantly correlated with each other $(\mathrm{p}=0.035)$, Pearson's product-moment correlation was weak $(r=0.34)$, indicating that GDF-15 may not directly influence the hepcidin level and may thus - by itself - not qualify for indicating the inter-individual differences in regulation of iron homeostasis.

\section{Discussion}

Oral iron supplementation is generally recommended to blood donors, based on a randomized, placebo-controlled, double-blind study demonstrating that $20 \mathrm{mg}$ of elemental iron per day can adequately compensate for iron loss in males and females who donate whole blood up to 4 (females) or 6 times per year (males). Nevertheless, there are significant inter-individual difference in intestinal iron uptake [7]. Furthermore, Mast and colleagues [28] identified a subgroup of blood donors who do not develop SID despite repeated and frequent blood donations without any oral iron supplementation. This subpopulation is heterozygous for the H63D mutation in HFE and shows significantly low levels of hepcidin and decreased hepcidin/ferritin quotients compared to other individuals. Thus, we tried to find an algorithm which can help to better distinguish between donors who benefit from iron supplementation and those who rather need longer interdonation intervals.

Testing a broad spectrum of parameters we wanted i) to assure the reliability of our data by confirming that the subjects in our study group did not suffer from (subclinical) infections, inflammations, and kidney or liver diseases interfering with measured parameters, ii) to assess erythropoiesis which also impacts iron homeostasis, and iii) to maximize the chance to define an early factor or algorithm which can help to distinguish between donor subpopulations.

Not being sure about the width of the diagnostic window for such an early factor/algorithm, we decided to ask our study subjects not to take any iron supplements which could have narrowed or even closed the possible time window to detect any inter-individual differences in tested parameters. Nevertheless, a prospective study comparing donors with and without oral iron supplementation needs to be performed in order to calculate the predictive value of the hepcidin/ferritin quotient.

Our study was performed on male donors only in order to exclude biases such as menstrual bleeding or different relative blood loss after a donation due to discrepancies in average body weight or dietary habits between the two genders. However, iron homeostasis seems to differ between men and women [7] emphasizing the need for further studies including both genders.

The logarithm of the ratio of the soluble Trf-R to ferritin concentration $(\log [\mathrm{TfR} / \mathrm{F}])$, which was shown to have a highly linear correlation to body storage iron, is currently the most precise measure of body storage iron available. Thus we also checked for this parameter and confirmed the inability of donors to spontaneously recover from iron loss following blood donation (fig. 1).

Our observation of a weak correlation between GDF-15 and hepcidin levels was consistent with published data [26, 29], indicating that GDF-15 may not directly influence hepcidin levels [30, 31].

As hepcidin appears to integrate erythropoietic and iron-loading signals, clinical measurement of hepcidin (together with the hepcidin-ferritin ratio) may become a useful indicator of erythropoiesis and iron kinetics

Detection of SID in blood donors is important not only to prevent donors from becoming anemic after subsequent blood donations but also because of the fact that iron deficiency affects various 
other cellular processes, given that iron is an important constituent of a series of enzymes. For example, previous studies suggested that iron deficiency affects DNA synthesis [32,33], the immune system, and energy metabolism through impaired mitochondrial electron transport $[35,36]$.

Furthermore, donors with SID are more prone to develop donation-induced $\mathrm{Hb}$ decrease possibly leading to subsequent experience of deferral from blood donation due to non-eligible $\mathrm{Hb}$ values. Considering that about $40 \%$ of repeat blood donors do not reappear when experiencing a deferral it is of great importance to recognize SID early enough to avoid subsequent $\mathrm{Hb}$-based deferral and also to establish an algorithm to make more individualized recommendations for oral iron uptake which - by itself - would also increase blood donors' satisfaction and trust in medical surveillance performed by blood donation centers.

We therefore suggest ferritin measurements at the time of first blood donation and once a year for donors with more than 4 donations within 12 months along with a general recommendation for oral iron uptake based on data from Radtke et al. [27] who have already demonstrated that most (but not all) blood donors benefit from a daily oral iron supplementation of $20 \mathrm{mg}$. Nevertheless, individualized recommendations (particularly for repeat donors) are advisable as a long-term strategy.

Based on our data, we believe that the hepcidin/ferritin quotient may provide an algorithm helping to decide if a donor may benefit from oral iron supplementation or rather from a longer interdonation interval. Assuming that individual hepcidin responsiveness to iron loss does not change, we suggest calculating hepcidin/ferritin quotient for repeat donors (just once) at the time of their 4th donation. This would be a reasonable strategy considering also economic issues.

\section{Disclosure Statement}

The authors certify that they have no affiliation with or financial involvement in any organization or entity with a direct financial interest in the subject matter or materials discussed in this manuscript

\section{References}

1 Chen PP, Short TG, Leung DH, Oh TE: A clinical evaluation of the Hemocue haemoglobinometer using capillary, venous and arterial samples. Anaesth Intensive Care 1992;20:497-500.

2 James V, Jones KF, Turner EM, Sokol RJ: Statistical analysis of inappropriate results from current $\mathrm{Hb}$ screening methods for blood donors. Transfusion 2003;43:400-404.

3 Lotfi R, Wernet D, Starke U, Northoff H, Cassens U: A noninvasive strategy for screening prospective blood donors for anemia. Transfusion 2005;45:1585-1592.

4 Rippmann CE, Nett PC, Popovic D, Seifert B, Pasch T, Spahn DR: Hemocue, an accurate bedside method of hemoglobin measurement? J Clin Monit 1997;13:373377.

5 Simon TL: Iron, iron everywhere but not enough to donate. Transfusion 2002;42:664.

6 Baart AM, van Noord PA, Vergouwe Y, Moons KG Swinkels DW, Wiegerinck ET, de Kort WL, Atsma F: High prevalence of subclinical iron deficiency in whole blood donors not deferred for low hemoglobin. Transfusion 2013;53:1670-1677.

7 Garry PJ, Koehler KM, Simon TL: Iron stores and iron absorption: effects of repeated blood donations. Am J Clin Nutr 1995;62:611-620.

8 Jelkmann W: Regulation of erythropoietin production. J Physiol 2011;589:1251-1258.

9 Gardenghi S, Marongiu MF, Ramos P, Guy E, Breda L, Chadburn A, Liu Y, Amariglio N, Rechavi G, Rachmilewitz EA, Breuer W, Cabantchik ZI, Wrighting DM, Andrews NC, de SM, Giardina PJ, Grady RW, Rivella S: Ineffective erythropoiesis in beta-thalassemia is characterized by increased iron absorption mediated by down-regulation of hepcidin and up-regulation of ferroportin. Blood 2007;109:5027-5035.

10 Nemeth E, Tuttle MS, Powelson J, Vaughn MB, Donovan A, Ward DM, Ganz T, Kaplan J: Hepcidin regulates cellular iron efflux by binding to ferroportin and induc ing its internalization. Science 2004;306:2090-2093.
Talbot NP, Lakhal S, Smith TG, Privat C, Nickol AH, Rivera-Ch M, Leon-Velarde F, Dorrington KL, Mole DR, Robbins PA: Regulation of hepcidin expression at high altitude. Blood 2012;119:857-860.

12 Pasricha SR, McQuilten Z, Westerman M, Keller A, Nemeth E, Ganz T, Wood E: Serum hepcidin as a diagnostic test of iron deficiency in premenopausal female blood donors. Haematologica 2011;96:1099-1105.

13 Nemeth E, Valore EV, Territo M, Schiller G, Lichtenstein A, Ganz T: Hepcidin, a putative mediator of anemia of inflammation, is a type II acute-phase protein. Blood 2003;101:2461-2463.

14 Kemna E, Pickkers P, Nemeth E, van der Hoeven H, Swinkels D: Time-course analysis of hepcidin, serum iron, and plasma cytokine levels in humans injected with LPS. Blood 2005;106:1864-1866.

15 Ganz T, Olbina G, Girelli D, Nemeth E, Westerman M: Immunoassay for human serum hepcidin. Blood 2008; 112:4292-4297.

16 Zaritsky J, Young B, Wang HJ, Westerman M, Olbina G, Nemeth E, Ganz T, Rivera S, Nissenson AR, Salusky IB: Hepcidin - a potential novel biomarker for iron status in chronic kidney disease. Clin J Am Soc Nephrol 2009;4:1051-1056.

17 Ginzburg Y, Rivella S: Beta-thalassemia: a model for elucidating the dynamic regulation of ineffective erythropoiesis and iron metabolism. Blood 2011;118:43214330.

18 Ashby DR, Gale DP, Busbridge M, Murphy KG, Duncan ND, Cairns TD, Taube DH, Bloom SR, Tam FW, Chapman R, Maxwell PH, Choi P: Erythropoietin administration in humans causes a marked and prolonged reduction in circulating hepcidin. Haematologica 2010;95:505-508.

19 Pak M, Lopez MA, Gabayan V, Ganz T, Rivera S: Suppression of hepcidin during anemia requires erythropoietic activity. Blood 2006;108:3730-3735.

20 Kautz L, Jung G, Valore EV, Rivella S, Nemeth E, Ganz T: Identification of erythroferrone as an erythroid regulator of iron metabolism. Nat Genet 2014; 46:678-684.
1 Tanno T, Bhanu NV, Oneal PA, Goh SH, Staker P, Lee YT, Moroney JW, Reed CH, Luban NL, Wang RH, Eling TE, Childs R, Ganz T, Leitman SF, Fucharoen S, Miller JL: High levels of GDF15 in thalassemia suppress expression of the iron regulatory protein hepcidin. Nat Med 2007;13:1096-1101

22 Tanno T, Noel P, Miller JL: Growth differentiation factor 15 in erythroid health and disease. Curr Opin Hematol 2010;17:184-190.

23 Tanno T, Porayette P, Sripichai O, Noh SJ, Byrnes C, Bhupatiraju A, Lee YT, Goodnough JB, Harandi O, Ganz T, Paulson RF, Miller JL: Identification of TWSG1 as a second novel erythroid regulator of hepcidin expression in murine and human cells. Blood 2009; 114:181-186.

24 Beguin Y: Soluble transferrin receptor for the evaluation of erythropoiesis and iron status. Clin Chim Acta 2003;329:9-22.

25 Huebers HA, Beguin Y, Pootrakul P, Einspahr D, Finch CA: Intact transferrin receptors in human plasma and their relation to erythropoiesis. Blood 1990;75:102-107.

26 Mast AE, Schlumpf KS, Wright DJ, Custer B, Spencer B, Murphy EL, Simon TL: Demographic correlates of low hemoglobin deferral among prospective whole blood donors. Transfusion 2010;50:1794-1802.

27 Radtke H, Tegtmeier J, Rocker L, Salama A, Kiesewetter H: Daily doses of $20 \mathrm{mg}$ of elemental iron compensate for iron loss in regular blood donors: a randomized, double-blind, placebo-controlled study. Transfusion 2004;44:1427-1432.

28 Mast AE, Foster TM, Pinder HL, Beczkiewicz CA, Bellissimo DB, Murphy AT, Kovacevic S, Wroblewski VJ, Witcher DR: Behavioral, biochemical, and genetic analysis of iron metabolism in high-intensity blood donors. Transfusion 2008;48:2197-2204.

29 Tarkun P, Mehtap O, Atesoglu EB, Geduk A, Musul MM, Hacihanefioglu A: Serum hepcidin and growth differentiation factor-15 (GDF-15) levels in polycythemia vera and essential thrombocythemia. Eur J Haematol 2013;91:228-235. 
30 Casanovas G, Spasic MV, Casu C, Rivella S, Strelau J, Unsicker K, Muckenthaler MU: The murine growth differentiation factor 15 is not essential for systemic iron homeostasis in phlebotomized mice. Haematologica 2013;98:444-447.

-31 Tanno T, Rabel A, Lee YT, Yau YY, Leitman SF, Mille JL: Expression of growth differentiation factor 15 is not elevated in individuals with iron deficiency secondary to volunteer blood donation. Transfusion 2010;50: 1532-1535.

32 Hoffbrand AV, Ganeshaguru K, Hooton JW, Tattersal MH: Effect of iron deficiency and desferrioxamine on DNA synthesis in human cells. Br J Haematol 1976;33: 517-526.
3 Furukawa T, Naitoh Y, Kohno H, Tokunaga R, Taketani S: Iron deprivation decreases ribonucleotide reductase activity and DNA synthesis. Life Sci 1992;50: 2059-2065.

34 Ward RJ, Crichton RR, Taylor DL, Della CL, Srai SK, Dexter DT: Iron and the immune system. J Neural Transm 2011;118:315-328.

35 Finch CA, Miller LR, Inamdar AR, Person R, Seiler K, Mackler B: Iron deficiency in the rat. Physiological and biochemical studies of muscle dysfunction. J Clin Invest 1976;58:447-453.
36 Ackrell BA, Maguire JJ, Dallman PR, Kearney EB: Effect of iron deficiency on succinate- and NADHubiquinone oxidoreductases in skeletal muscle mitochondria. J Biol Chem 1984;259:10053-10059.

7 Hillgrove T, Moore V, Doherty K, Ryan P: The impact of temporary deferral due to low hemoglobin: future return, time to return, and frequency of subsequent donation. Transfusion 2011;51:539-547. 